\title{
Investigation of flow through a computationally-generated packed column using CFD and Additive Layer Manufacturing.
}

\author{
M.J Baker, S. Daniels, P.G Young, G.R Tabor ${ }^{1}$ \\ College of Engineering, Maths and Physical Sciences (CEMPS), Harrison \\ Building, University of Exeter, North Park Road, Exeter EX4 4QF, UK
}

\begin{abstract}
When analysing packed beds using CFD approaches, producing an accurate geometry is often challenging. Often a computational model is produced from non-invasive imaging of the packed bed using $3 \mathrm{~d}$ MRI or $\mu$-CT. This work pioneers the exact reverse of this, by creating a physical bed from the computational model using additive layer manufacturing (ALM). The paper focuses on both experimental and computational analysis of packed columns of spheres. A STL file is generated of a packed column formed using a Monte-Carlo packing algorithm, and this is meshed and analysed using computational fluid dynamics. In addition to this, a physical model is created using ALM on a $3 \mathrm{~d}$ printer. This allows us to analyse the identical bed geometry both computationally and experimentally and compare the two. Pressure drop and flow patterns are analysed within the bed in detail.
\end{abstract}

Key words: Packed Bed, Pressure drop,Porous media, Fluid mechanics, Computational fluid dynamics, Simulation

\section{Introduction}

Packed columns and packed beds are used in industry for a variety of operations including absorption, stripping and catalysis (Perry and Green, 1997). Hence there is significant interest in analysing their behaviour (Sahimi, 1994), particularly in regard to understanding the physical processes involved (Adler, 1992). A packed bed is a large structure filled with a packing material, which can be random in size and shape, such as gravel or charcoal, or be composed

$\overline{1}$ Corresponding author.Email: g.r.tabor@ex.ac.uk 
of uniform shapes such as spheres or cylinders. When a fluid passes through a packed bed, it percolates through a network of voids or channels and interacts with the bed particles, creating a drop in pressure. Thus a packed bed is generally characterised by a number of key parameters such as porosity, particle size and bed depth. In the case of catalysts and other industrial processes, bed surface area may also be a key parameter in achieving maximum fluid-to-particle interaction. A packed column is a special case where the bed is contained in a tube or other narrow cylindrical vessel. The flow in packed columns is more complex both due to boundary effects at the walls of the vessel, and also because it upsets the packing of the particles within the column.

Traditionally, the principal method for examining the flow through a packed bed has been through experimentation, but with the development of computer analysis has come the possibility of applying Computational Fluid Dynamics (CFD) to analyse the large and small scale flow through these structures (see Dixon et al., 2013, for a review). Typically in CFD the domain of interest is divided into numerous small cells forming a mesh (Finite Volume technique) or elements forming a grid (Finite Element approach) on which the equations of motion for the fluid can be discretised and solved numerically; for packed beds, Lattice Boltzmann techniques, discretising the Boltzmann transport equation on a regular grid, have also been applied with some success. Constructing an adequate mesh for a complex domain is a challenging task, but at the same time the accuracy of the solution or even the possibility of generating a solution can depend on the quality of the mesh. In the early application of CFD to the analysis of packed beds, limited computing resources dictated the analysis of simplified geometries, and most often, CFD analysis was applied to unit cell geometries which it was hoped could be considered to be representative of a full but structured bed geometry. Today, with increased computational resources, random packed beds comprising hundreds of particles can be attempted (e.g. Atmakidis and Kenig, 2009; Augier et al., 2010; Eppinger et al., 2011).

Creation of the geometry and mesh for such a complex case is however still one of the primary obstacles to using CFD in this manner. The task involves specifying the geometry of the complex domain representing the space around the particles to a high level of geometric fidelity, and discretising this space into millions of computational elements (numbers of computational elements currently employed range from $2-30$ million) whilst ensuring that mesh quality indicators such as $y^{+}$values and skewness indicators do not exceed acceptable values. Systematic development of meshing strategies can be informed from consideration of modelling the flow around a single particle (Dixon et al., 2011) but this can be of limited use for multiple particle simulations. Contact points between the particles are particularly problematic as regards mesh quality, with the particle surfaces meeting tangentially, leading to pathological cell shapes and the need for extreme numerical correction. Alternatively, 
various methodologies for adapting the geometry around contact points have been considered (Dixon et al., 2013).

One approach to geometry and mesh generation is to develop the geometry using CAD which can then be passed to a standard automatic mesh generator. This can be achieved for packing regimes such as simple cubic, close hexagonal etc where the particle locations are predictable and can be determined from simple geometry. For an experimental validation, a structured bed or a simple unit cell can be easily be constructed for direct comparison and verification of computational data (Tobis, 2002). To generate a computational random unstructured bed from scratch is more challenging and usually requires the use of a procedure such as a Monte-Carlo algorithm to generate random particle locations (e.g. Atmakidis and Kenig, 2009; Augier et al., 2010; Eppinger et al., 2011). The alternative to this is to start with an actual physical packed bed and to try to construct the geometry and mesh to match it (Mantle et al., 2001; Sullivan et al., 2005; Baker et al., 2011). This can for example be accomplished using techniques of Image Based Meshing (IBM) developed initially for biomedical simulation, where there is a very similar problem (developing the mesh for a problem where the geometry is very complex and frequently unknown). A non-invasive technique such as magnetic resonance imaging (MRI) or computed tomography (CT) can be applied to scan a particular experimental bed. A stack of greyscale images is produced which can be used as a template to generate a mesh. This can be accomplished through several different routes, including methods referred to as 'voxel-based' approaches. In this approach, the stacks of pixels are regarded as a regular array of volumes - the voxels. A group of voxels can be identified to represent the domain of interest, a process referred to as segmentation, and the set of voxels so identified is referred to as a mask. The marching cubes algorithm can be used to generate a hexahedral base mesh which can then be truncated and smoothed to form the volumetric mesh in one operation. This voxel-based method is used in the commercial code ScanIP which will be used in this work.

Using IBM methods the reproduced computational geometry may be considered to be essentially the same as the experimental bed. However, fidelity issues often arise associated with the scan resolution and artefacts. For instance, unless the initial scans are very binary, with a sharp contrast between particle and non-particle, there may be a degree of ambiguity as to where exactly the domain boundary lies, affecting the volume of the flow domain and thus the overall porosity. Also, particles have been shown to merge at the contact points, referred to as necking, causing a reduction in geometric faithfulness (Baker et al., 2011). This has also been demonstrated with other meshing techniques (see Ookawara et al. (2007), who refer to this as "bridging"). In the case of relatively low aspect ratio beds, this phenomenon can affect the porosity quite considerably leading to a discrepancy between computational and experimental beds. There is one advantage to this phenomenon; the contact 
points are now represented by a neck of solid material rather than an infinitesimally small point, reducing the likelihood of generating highly skewed cells often produced around particle contacts in the discretization process.

Another, related issue is the problem of validation. CFD can be highly accurate but it is ultimately a numerical approximation to the actual flow. Additionally, the CFD modelling process involves the interaction of a large number of complex physical, mathematical and numerical models, inadequacies or errors in any of which can lead to inaccurate and sometimes misleading results. CFD accordingly has to be validated by comparison with experimental results, preferably carried out on the same or possibly equivalent geometries. The two issues (experimental validation and mesh generation) are related as taking a particular approach to one can constrain or specify the approach taken to the other. Validation has typically been carried out by comparison with well recognised correlations such as that of Eisfeld and Schnitzlein (2001); but these correlations are composite relations constructed from a spread of experimental data, not necessarily matching the computational bed in all properties (Augier et al., 2010). For a more precise experimental comparison/validation, an equivalent packed bed can be constructed with the same geometric properties, such as particle diameter, porosity, depth and aspect ratio. One advantage of scanning techniques for computational model development is that an actual physical bed exists which can be used for the experiment. If the bed was initially designed computationally, then the experimental bed may only be similar; the geometric properties may indeed be the same, however the flow paths and internal tortuosity may vary quite considerably. The comparison of computational data with experimental equivalent beds has shown to produce good results for global properties, such as the pressure drop caused by the media; it has also shown to be suitable for verifying computational results (Baker and Tabor, 2010). There is still the question of whether the simulation accurately reproduces the actual internal flow structures, for which detailed flow measurements within the bed would be needed (for instance through MRI velocimetry, e.g. (Robbins et al., 2012)).

In this work we develop a third alternative, effectively reversing the above processes by creating a computational model of the domain and then using Additive Layer Manufacturing techniques to create a physical model for testing. To start with, a Monte-Carlo particle packing code MacroPac is used to generate a randomly packed, volume-bidistributed bed of $10 \mathrm{~mm}$ and $16 \mathrm{~mm}$ spheres, in a similar approach to that used in Baker and Tabor (2010). The sphere sizes were chosen to promote packing and thus increase the number of contact points. From MacroPac the particle location coordinates and diameters are exported to a text file where a simple $\mathrm{C}$ program converts coordinate locations into a STL (STereoLithography) file. Meshing software (ScanIP, Simpleware, Exeter, UK) is used to convert the STL CAD geometry into a finite volume mesh for CFD analysis using the commercial CFD code Fluent. At 
the same time the STL file created during this process is exported to an ALM rapid protyping machine for manufacture; a $3 \mathrm{~d}$ printer using the fused deposition method. The resulting physical structure can be used for experimental analysis, in this case pressure drop measurements using the experimental apparatus described in Baker and Tabor (2010). Thus, this chain of bed creation ensures an experimental packed bed for analysis which is geometrically faithful to the computational bed.

The structure of the paper is as follows. In the next section we will describe the experimental methodology used, focussing on the use of additive layer manufacture to create the physical bed, but also providing an overview of the computational methods used to generate the virtual bed, and the experiments being performed. The following section will describe the numerical flow analysis undertaken. Section 5 will present results and comparisons between physical and experimental analysis, and the final section will draw conclusions regarding the work.

\section{Experimental methodology}

\subsection{Computational Packing of Spheres}

In this work, the particle packing simulation program MacroPac (Intelligensys, UK) was used to generate packed beds of spheres using a Monte Carlo method to ensure the densest possible packing by simulated shaking of the column. In this work the spheres are bi-distributed, weighted $50: 50$ by volume with diameters of $16 \mathrm{~mm}$ and $10 \mathrm{~mm}$. Burke and Plummer (1928) and Gupte (1970) have proved by experiment that packed beds of mixed spheres show a higher drop in pressure compared with beds of mono-sized spheres and as a result they can not simply be treated in the same way by using the equivalent diameter. Here, the basic underlying physics used by MacroPac is explained as follows. The probability that a particle moves in the $\mathrm{z}$-axis is

$$
p=e^{-\frac{\Delta E}{R \cdot T}}
$$

$\Delta E$ is the change in potential energy when the object moves against a force in the $-z$ direction. We know change in potential energy is given as

$$
\Delta E=m g \Delta h
$$

In the simulation the probability that a particle moves up 


$$
p=e^{m_{r} \Delta h_{r} K_{f}}
$$

where $m_{r}$ is the unit of mass and $\Delta h_{r}$ is the distance moved. $m_{r}$ and $\Delta h_{r}$ are defined by the objects mass density relationship, $m=\rho V$ and boundary dimension parameters. $K_{f}$ is the kinetic factor given as

$$
K_{f}=\frac{m_{f} h_{f} g}{R \cdot T}
$$

The kinetic factor is also related to the amount of shaking; more shaking will produce a lower kinetic factor as more particles may move up due to the shake.

Having generated the computational packed bed, an STL file is created from a simple, purpose written $\mathrm{C}$ program from the raw coordinate data and particle diameters which can be used for constructing the physical bed through ALM (below) and CFD simulation (section 3.1).

\subsection{Additive Layer Manufacturing}

Rapid prototyping was first developed in the late 1980's and its primary use at the time was to create prototypes of components to be manufactured. The field has advanced considerably in both speed of manufacture and component quality, to the extent that it is no longer just limited to prototyping but also in direct manufacture, and so is now referred to by practitioners as Additive Layer Manufacturing. Various ALM methods exist, depending on what processes are used to form successive layers and fuse them onto the earlier layers. In all cases, the geometry to be formed (usually described as an STL file) is sectioned computationally into a sequence of slices to be manufactured. Then, in selective laser sintering (SLS) successive layers of powder (metal or plastic) are laid down on the object and a high powered laser used to selectively fuse the particles together in the specified areas. Similar techniques include stereolithography (a photosetting liquid polymer and a high powered UV source are used) and electron beam melting (an electron beam in a vacuum is used to fuse a metal powder). The main alternative technology is fused deposition modelling (FDM) or 3d printing (3DP) in which a fast-setting liquid is ejected from a movable head scanning across the build plane. For this work we adopt a 3DP/FDM approach due to its speed of manufacture and cost. The model is manufactured using the thermoplastic Acrylonitrile butadiene styrene (ABS). Whilst being constructed the ABS structure is held together with a temporary structure which is water soluble. On build completion the model is left to soak in detergent to allow the temporary structure to dissolve. 
When a FDM approach is adopted the particles exhibit a slight roughness in the form of horizontal bands. There is a certain amount of controversy over the influence of particle roughness on the pressure drop. We know from literature that many of the classical correlations are based on the assumption that flow through the bed is equivalent to flow through a set of tubes (with corrections for issues such as tortuosity). (Burke and Plummer, 1928; Carman, 1937; Ergun and Orning, 1949) and therefore should obey the same assumptions as simple pipe flow; the rougher the wall and/or longer the pipe (more tortuous in the case of packed beds) the greater the drop in pressure. Leva and Grummer (1947); Leva et al. (1951) and Dullien (1979) suggest there is an increase in pressure drop due to the particles roughness. In contrast, others observe little or no effect (see Fand et al., 1972; Brownell et al., 1950). Eisfeld (1999); Eisfeld and Schnitzlein (2001) analysed the research of Leva et al. (1951) and Dullien (1979) and concluded that their observations are most probably due to experimental error or a misinterpretation of data suggesting that the drop in pressure is not affected by particle roughness or at least that this parameter has an unmeasurable effect.

We have already discussed the effect of the necking due to additional voxels of particles and the detrimental effect it has on the fidelity of a computational model. Here, the effect of the necking is used as an advantage. Without the phenomenon of necking the particles the physical structure would be statically unstable and liable to fall apart. Although actual packed beds do not display particle necking, here we are interested in replication of the exact geometry with fidelity rather than reproducing all the details of a real life packed bed. This method is therefore completely geometrically faithful to the mesh in respect to geometry, particle size etc. The only physical difference between the experimental bed and computational is the fine horizontal banding produced by the RP resolution. but this banding is considered sufficiently small to be considered as a surface finish rather than a variance in the geometry. The computational and printed beds are compared in figure 1.

\subsection{Experimental Setup}

The experimental setup is shown in figure 2. The apparatus is based on that of (Tóbis, 2000), as described in (Baker and Tabor, 2010). The manufactured bed is inserted into a perspex tube $(D=100 \mathrm{~mm})$ with pressure tappings before and after the bed. The bed is $140 \mathrm{~mm}$ in height and consists of 670 $16 \mathrm{~mm}$ and $10 \mathrm{~mm}$ spheres weighted $50: 50$ by volume. Air is forced through the bed by means of a centrifugal pump. Volumetric flow rate $Q$, and hence average velocity $U$, was determined by measuring differential pressure $(\Delta P)$ across a plate orifice manufactured from Perspex and previously calibrated. The plate orifice method was chosen over that of a Pitot static tube, because the Pitot 

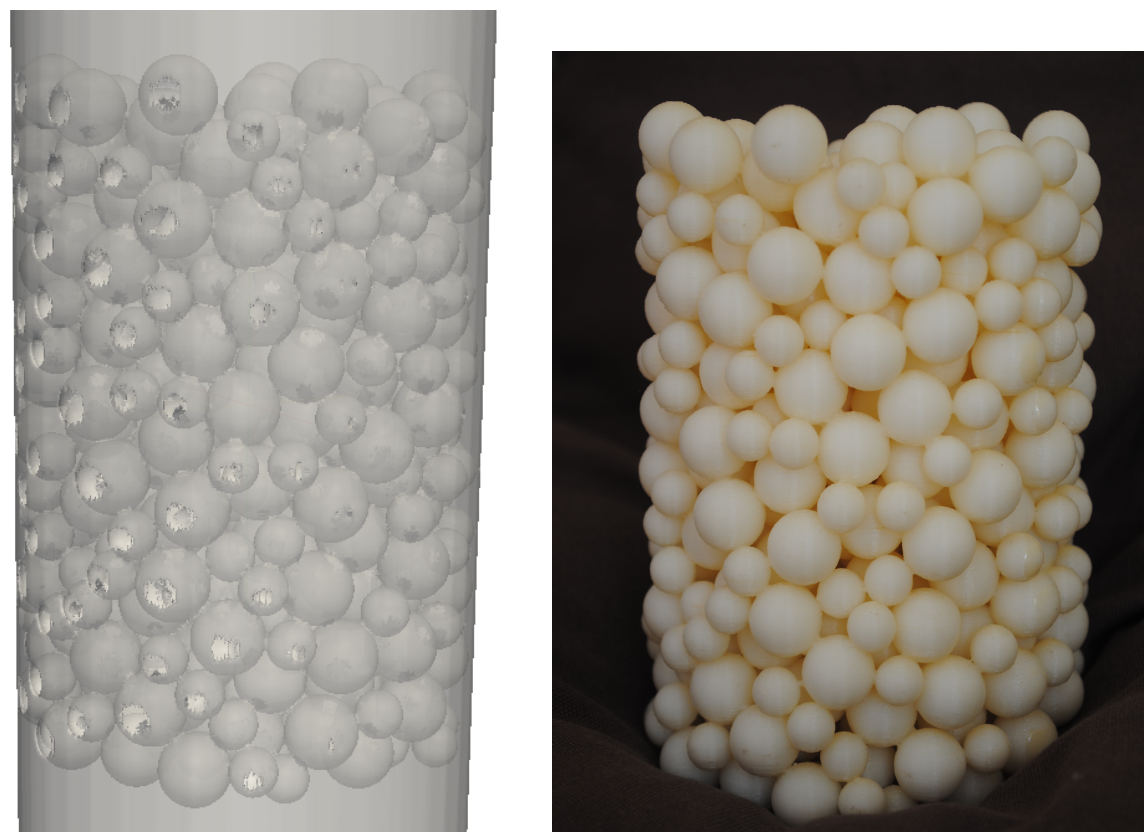

Fig. 1. Photograph of computationally generated bed (Left) and physical reconstruction through ALM bed (Right).

static tube only determines the velocity at its immediate location, meaning that a full flow profile using this method would require many readings to be taken in different locations across the pipe section, then averaged to reduce error. Experimental pressure drop in this work was calculated by means of a differential micro-manometer attached to a data logger. The data logger readings were averaged to reduce error from small fluctuations in pressure due to turbulence.

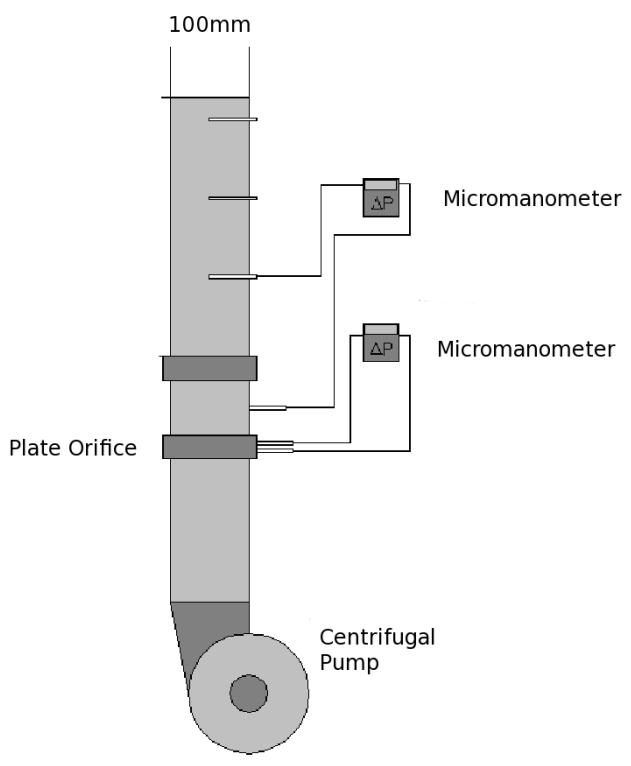

Fig. 2. Experimental rig. 


\section{Computational analysis}

The second part of the work described in this paper is the computational analysis. Two commercial packages are used for this work. The actual computational fluid dynamical analysis is accomplished with the finite volume code Fluent, based on finite volume meshes created using ScanIP, ScanFE and ScanCAD (Simpleware Ltd). Details of these codes and their use are provided in this section.

\subsection{Computational Fluid Dynamics (CFD)}

Computational fluid dynamics is the use of computers to solve problems associated with fluid motion. This is achieved by a computer generating an approximate solution to a set of partial differential equations; the Navier-Stokes equations or for turbulent flow, equations derived from these. The NavierStokes equations form a set of non-linear, second order, partial differential equations which encompass the fundamental principles of conservation of mass and momentum for the fluid. For a laminar flow, the equations being solved are as follows :

$$
\begin{aligned}
\nabla \cdot \vec{u} & =0 \\
\frac{\partial \vec{u}}{\partial t}+\nabla \cdot \vec{u} \vec{u} & =-\frac{1}{\rho} \nabla p+\nu \nabla^{2} \vec{u}
\end{aligned}
$$

The question of whether to use a turbulence model for this case is a complex one. In a previous paper (Baker et al., 2011) we argued that the flow in a packed bed was likely transitional to turbulent but affected by the complex geometry of the domain; however the small scale of the geometry also dictates a mesh fine enough to resolve most of the turbulent scales. In that work we also demonstrated that in practice the turbulence model had a minimal effect on the final solution in terms of the drop in pressure. Thus in the current work we have decided to present results from laminar flow solution alone.

We solve the governing equations (5) using the finite volume approach. In this, the domain of interest is divided into numerous small cells, and the governing equations integrated across the volume of each cell. Gauss' theorem is used to convert the spatial derivative term $\nabla \cdot(\vec{u} \vec{u})$ into a surface integral of the flux across the faces bounding the cell, which converts the governing equations into a set of difference equations which can be solved numerically. For this we use the commercial CFD code Fluent; interpolation from cell centre to cell face is carried out using 2nd order upwind differencing for the momentum equation, and the non-linear nature of the equations is treated iteratively, using PISO for transient calculations and SIMPLE for steady state calculations. To ensure 
stability, simulations were run initially using a steady state solver and then using this as a basis progressing to an unsteady solver. Convergence to a solution was judged based on the mass flow rate at the outlet of the domain.

\section{Computational domain}

In order to analyse the domain using CFD it is necessary to construct a finite volume mesh representing the geometry from the STL file. In theory, any mesher capable of importing STL files could be used for this task. However the domain is geometrically highly complex, and CAD-based meshers (such as Gambit) proved inadequate to the task. Cut-cell meshing approaches used in image based meshing are more robust however, so for this work the mesh was constructed using the image based meshing software ScanCAD, ScanIP and ScanFE in the same way as described in Baker et al. (2011). The ScanIP software operates using the voxel meshing method described above (section 1); however in this case instead of starting from a stack of scanned images, the companion software ScanCAD is used to generate a stack of voxels directly from the STL file. The voxelised bed can then be further processed in ScanIP as necessary. For this bed, the mesh contains 30,443, 307 cells, 62, 844, 838 faces and 6,679, 187 nodes.

It is plausible that the flow within the bed is being affected by the velocity profile upstream of the bed. In the experiment the bed is contained within a cylindrical pipe from the centrifugal pump which is long enough that the flow could exhibit a non-uniform profile when it reaches the bed. To examine the effect of this we investigated the effect of uniform and non-uniform flow profiles on the computed flow. The computational domain extended $20 \mathrm{~mm}$ upstream of the bed itself and a non-uniform velocity profile was imposed at this point, whose shape depended on the Reynolds number for the pipe flow. For a laminar flow $(\mathcal{R} e<2300$ based on pipe diameter) a parabolic profile was used :

$$
u=u_{0}\left(1-\frac{r^{2}}{R^{2}}\right)
$$

Turbulent $(\mathcal{R} e>4000)$ flow is considerably more complex and there are much higher losses in which case the profile is much flatter and decreases steeply at the wall region and is typically described by a $1 / 7$ power law profile :

$$
u=u_{0}\left(1-\frac{r}{R}\right)^{1 / 7}
$$

The alternative profile used was a simple flat profile. When presenting results with different velocity profiles an average velocity or volumetric flow rate is 
needed. In this work the plate orifice has given the volumetric flow rate $Q$, $\mathrm{m}^{3} / \mathrm{s}$ and hot-wire anemometry has given the centreline velocity $u_{\max }$. These can be related to the different flow profiles by integration.

\section{Results and discussion}

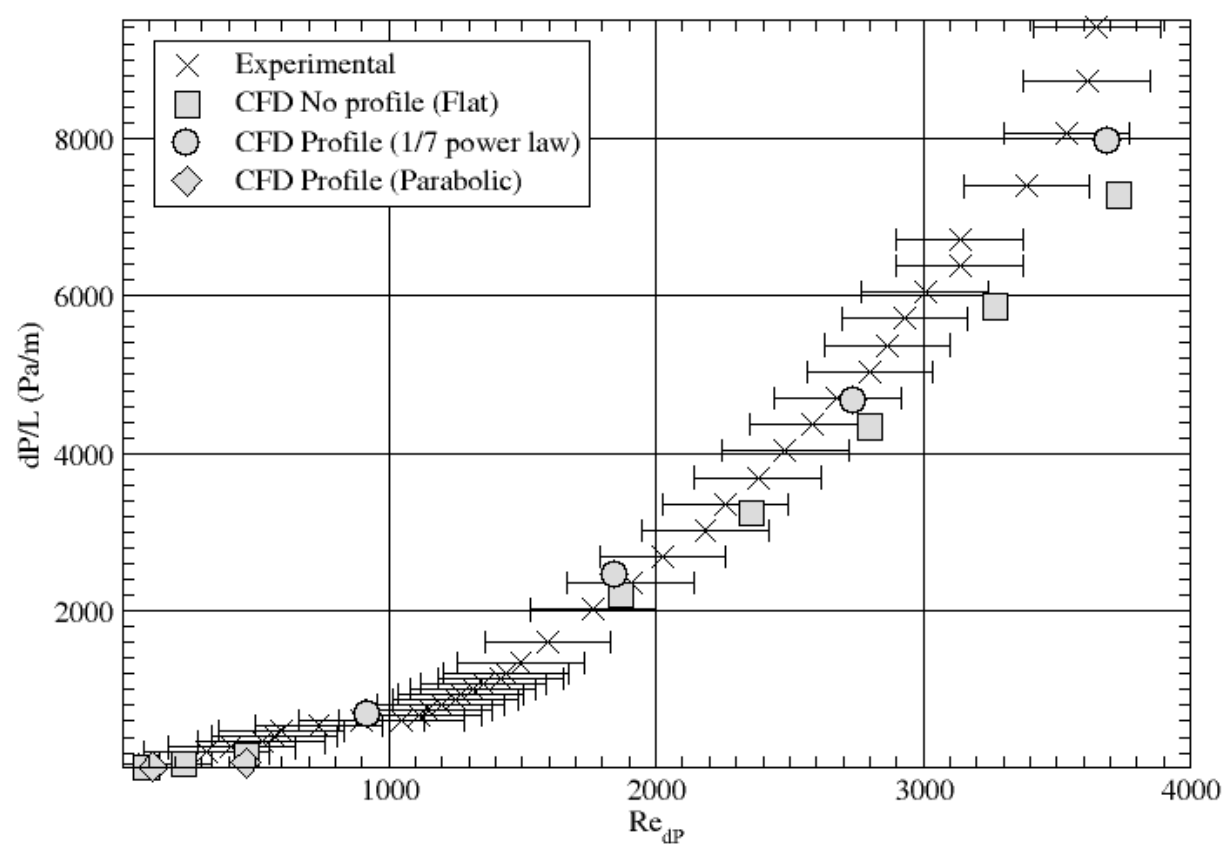

Fig. 3. Pressure drop per unit length as a function of Reynolds number for bi-distributed spheres of 16 and $10 \mathrm{~mm}$

Results from the various calculations and for the experimental measurement of pressure drop per unit length are plotted in figure 3 as a function of Reynolds number based on centreline velocity. Examining the flat profile results first, the agreement between experiment and CFD is very close for low flow rates, although a systematic limited divergence is apparent at the highest flow rates; this effect has been noted in earlier work (Baker et al., 2011). In the current work the deviation from the experimental work is $\sigma=0.61$ for the full range of Reynolds numbers; windowing to lower Reynolds numbers where the deviation is lower gives a value of $\sigma=0.06$. Including a non-flat inlet profile (parabolic for low Reynolds numbers, 1/7th power law for higher Reynolds numbers) makes a small difference, raising the pressure drop for the highest Reynolds numbers; in fact the turbulent profile is consistently above the results for the 


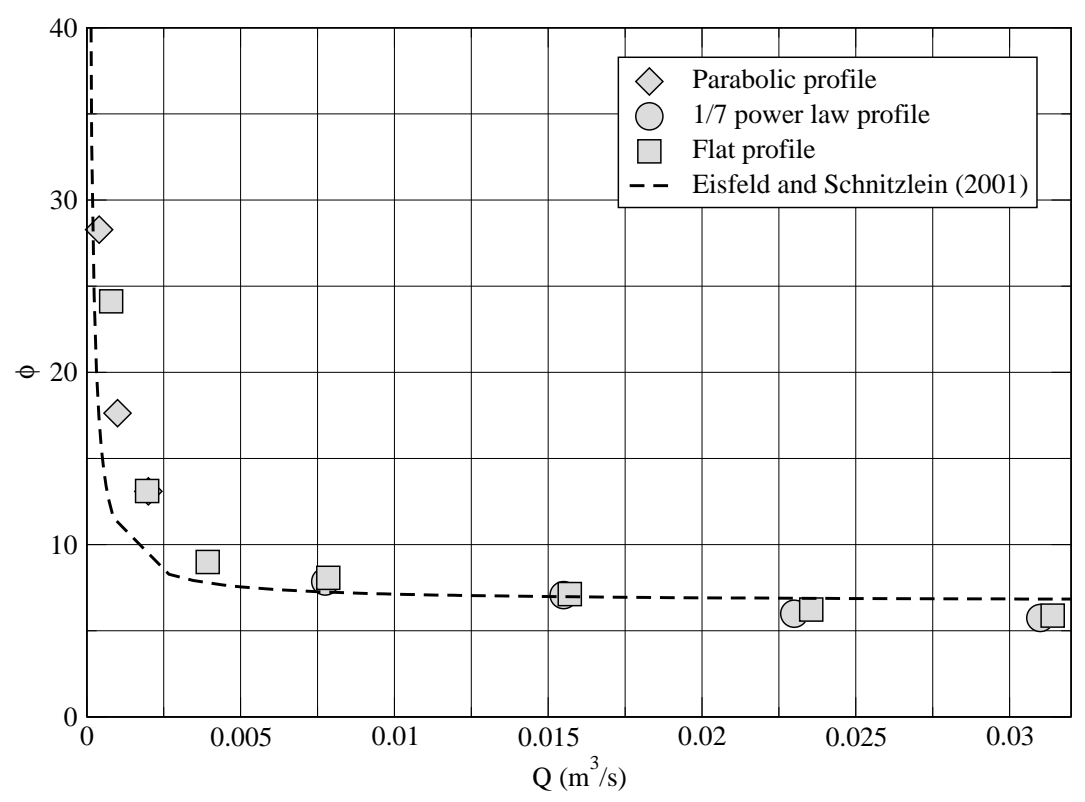

Fig. 4. Dimensionless pressure drop $\phi$ per unit length as a function of volumetric flow rate for the case of bi-distributed spheres of 16 and $10 \mathrm{~mm} ; 1 / 7$ th power law (triangles) and flat profile (circles) at inlet. Results are shown for flat inlet profile (squares) and laminar/turbulent inlet profiles (diamonds/circles).

flat profile, but the difference is only sizable for the highest Reynolds number case. For the lower Reynolds numbers the discrepancy is quite small. This is due to the bed geometry having a strong effect on the pressure gradient before the bed and distorting the velocity profile before the fluid reaches the media. This justifies the closeness of results to the experimental in earlier work without the use of a velocity profile for a fully turbulent regime. This result is emphasised by plotting dimensionless pressure drop $\phi$, given as

$$
\phi=\frac{\Delta P}{\rho_{0} U_{S}^{2}} \frac{d_{p}}{L}
$$

as shown in figure 4 .

Also shown in figure 4 is the correlation of Eisfeld and Schnitzlein (2001) which is an empirical correlation based on a sizable meta-dataset; a regression of over 2300 data points from 24 published articles generating a relation for cylinders and spheres. Many correlations exist to predict pressure drop through packed beds at low aspect ratios (Choi et al., 2008; Reichelt, 1972) but this correlation is regarded as one of the most promising, and it shows a very good correlation with our data. None of the studies used to develop the correlation of (Eisfeld and Schnitzlein, 2001) mention any effect of the velocity profile. Given 
that these were experimental studies it is probable that the velocity profile in the leadup to the bed was something between parabolic (ideal laminar flow) and $1 / 7$ power law (canonical turbulent flow profile), and since the profile was not being controlled for, the variance in inlet profiles between studies might account for some of the data spread observed. Based on the results demonstrated here however we would conclude that the inlet profile has little effect on the overall pressure drop across the bed.

Figure 5.a,b. depicts flow through the bed, taking random, representative streamwise and spanwise slices through the data for the flat inlet profile. The spanwise slice is quite close to the bed inlet plane. Preferential flow routes can easily be identified, particularly close to the walls. Figure 5.c. shows a closeup of the velocity vectors for a random section through the bed. We can see recirculation in stagnation zones and at the rear of the spheres in the pressure drag region which normally would be averaged out by the RANS equations when a turbulence model is implemented. A similar vortex structure behind spherical media has been observed by Dalman et al. (1986) using a computational approach and Yevseyev et al. (1991) using laser doppler anemometry. In a similar manner, Nakajo and Shigematsu measure turbulent stresses at similar locations within a regularly packed bed using Particle Tracking Velocimetry (a varient of standard PIV) (Nakajo and Shigematsu, 2011). Conversely, Tsotas (2002) reports no vortex shedding from particles around particle Reynolds numbers of $\mathcal{R} e=4780-7010$ which is in the range analysed here. However his experimental method relies on the use of hot-wire anemometers in the void space to detect unsteady flow behaviour. A hot wire anemometer utilises Newtons Law of cooling and the temperature difference to determine the velocity, and it is unlikely that the response time is fast enough to capture the highest frequency turbulent events in the turbulent spectrum. In addition, the bed analysed by Tsotas is based on a rhombahedral, structured packing and not a bi-distributed disordered pack, as here.

As mentioned earlier, much research has been devoted to the investigation of particle roughness. Leva et al. (1951) and Dullien (1979) observe and increased drop in pressure due the the particle roughness, whereas Fand et al. (1972) observe little or no effect. However Eisfeld and Schnitzlein (2001) analysed the research of Leva et al. (1951) and Dullien (1979) and concluded that their observations are most probably due to experimental error or a misinterpretation of data suggesting that the drop in pressure is not affected by particle roughness or at least that this parameter has an unmeasurable effect. Reinforcing the argument of Eisfeld and Schnitzlein (2001), the conflicting evidence could also be attributed to the level of the confidence involved in the studies. We know a packed bed can have the same dimensional parameters e.g porosity, particle diameter, length depth etc but can have a significantly different internal flow paths. This suggests that a bed of rough spheres cannot simply be packed and then compared to another pack of smooth spheres 
as the most influential parameter (the flow geometry) is being changed. In fact, to produce any credible level of confidence using this technique would require a population of hundreds if not thousands of packed bed samples, all with the same dimensional properties. In addition, the semi-empirical correlation devised by Eisfeld and Schnitzlein (2001) includes a graph in which over 2000 data points are plotted from various researchers. The work of Leva et al. (1951) is featured in this figure, and there is no discrepancy between the smooth particles of Reichelt (1972) and the rough particles of Leva et al. (1951). However, the spread of data is so vast (orders of magnitude) that any deviation due to particle friction is likely to go unnoticed and absorbed by experimental error and transient effects. In reality it is most probable that the particle friction is likely to be affecting the drop in pressure in some way, but this effect is likely to be so minimal and the effect of the geometry so predominantly influential that realistically the effect is not measurable or separable no matter how large the data set. The advantage of the approach described in our work is that actual beds can be physically recreated which are identical to the computational beds. This allows only one parameter to be changed keeping the geometry faithful. We know that the resolution can produce horizontal banding (roughness height of $0.1 \mathrm{~mm}$ ) and by changing this resolution beds with the same structure and different friction heights could be analysed. With a sufficiently large data set it is possible that conclusions could be made as to the the effect of particle roughness on the overall pressure drop, providing the effect could be physically measured. Improvements in ALM in the future may also make it possible to manufacture experimental beds with variations in roughness.

\section{Conclusions}

In this paper, computationally generated packed beds (generated using MacroPac) have been meshed using IBM techniques (ScanCAD, ScanFE), to create CFD models for bi-distributed beds. At the same time, ALM techniques have been used to synthesise a geometrically accurate physical bed for experimental investigation. Both experimental results carried out of the ALM bed and computational results have shown to match closely, particularly at the lower Reynolds range. As the Reynolds number increases the solver has a tendency to underpredict the pressure drop. The work has also investigated the application of the expected appropriate flow profiles to the computational models boundary conditions. Results have shown that that the velocity profile is having minimal effect on the pressure drop, except possibly at the highest Reynolds numbers. As a whole, this work has laid foundations for further work into the application of ALM in manufacturing disordered structures for comparison with CFD results. With the continual improvement in ALM currently seen, this 
a.

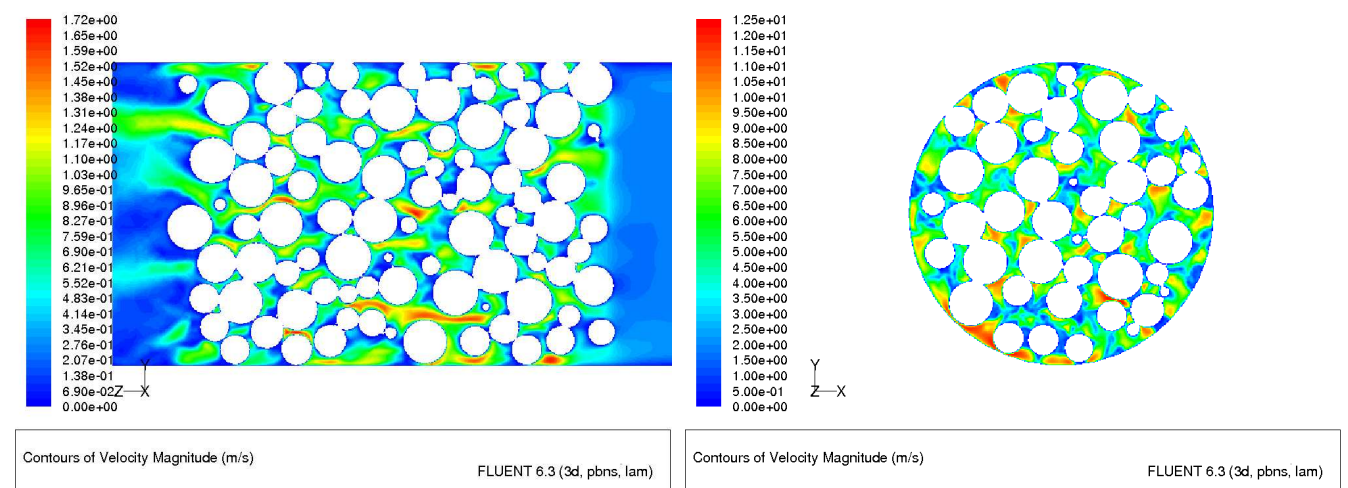

C.

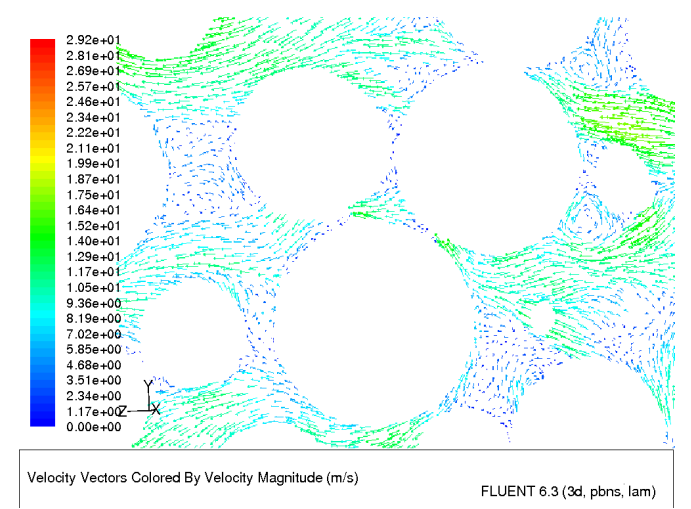

Fig. 5. Flow inside the bed at $3.0 \mathrm{~m} / \mathrm{s}$. a,b; flat velocity profile, c. closeup behind particles showing recirculation zones.

could represent a valuable new technique for exploring flow in packed beds, permitting, as we have shown here, validation of CFD on exact structures; increased printing will improve the ability to print finer structures, and the use of alternative materials such as metals could open the way to validating other physical processes such as heat exchange.

\section{References}

Adler, P. M., 1992. Porous Media: Geometry and Transport. ButterworthHeinemann, Stoneham, MA.

Atmakidis, T., Kenig, E. Y., 2009. Cfd-based analysis of the wall effect on the pressure drop in packed beds with moderate tube/particle diameter ratios in the laminar ow regime. Chem.Eng.J 155 (1-2), 404-410.

Augier, F., Idoux, F., Delenne, J. Y., 2010. Numerical simulations of transfer and transport properties inside packed beds of spherical particles. Chem.Eng.Sci. 65 (3), 1055 - 1064.

Baker, M., Young, P., Tabor, G. R., 2011. Image based meshing of packed beds 
of cylinders at low aspect ratios using 3d MRI coupled with computational fluid dynamics. Computers and Chemical Engineering 35, 1969 - 1977.

Baker, M. J., Tabor, G. R., 2010. Computational analysis of transitional airflow through packed columns of spheres using the finite volume technique. Computers and Chemical Engineering 34, 878 - 885.

Brownell, L. E., Dombrowski, H. S., Dickey, C. A., 1950. Pressure drop through porous media. part iv: New data and revised correlation. Chem.Eng.Prog. 46, $415-422$.

Burke, S. P., Plummer, W. B., 1928. Gas flow through packed columns. Industrial Engineering Chemistry 20, 1196-1200.

Carman, P. C., 1937. Fluid flow through a granular bed. Trans.Inst.Chem.Eng.London 15, $150-156$.

Choi, Y. S., Kim, S. J., Kim, D., 2008. A semi-empirical correlation for pressure drop in packed beds of spherical particles. Transp Porous Med 75 (2), 133 -149 .

Dalman, M. T., Merkin, J. H., McGreavy, C., 1986. Fluid flow and heat transfer past two spheres in a cylindrical tube. Computers and Fluids 14 (3), 267 $-281$.

Dixon, A. G., Nijemeisland, M., Stitt, E. H., 2013. Systematic mesh development for 3d CFD simulation of fixed beds: Contact points study. Computers and Chemical Engineering 48, 135 - 153.

Dixon, A. G., Taskin, M. E., Nijemeisland, M., Stitt, E. H., 2011. Systematic mesh development for 3d CFD simulation of fixed beds: Single sphere study. Computers and Chemical Engineering 35, 1171 - 1185.

Dullien, F. A. L., 1979. Porous Media: Fluid Transport and Flow Structure. Academic Press, New York.

Eisfeld, B., 1999. Pseudokontinuierliche modellierung der strömung in schüttschichtreaktoren. Ph.D. thesis, Brandenburgische Technische Universität Cottbus.

Eisfeld, B., Schnitzlein, K., 2001. The influence of confining walls on the pressure drop in packed beds. Chem.Eng.Sci. 56 (14), 4321 - 4329.

Eppinger, T., Seidler, K., Kraume, M., 2011. DEM-CFD simulations of fixed bed reactors with small tube to particle diameter ratios. Chem.Eng.J. 166, $324-331$.

Ergun, S., Orning, A., 1949. Fluid flow through randomly packed columns and fluidised beds. Ind.Eng. Chem 41, 1179 - 1184.

Fand, R. M., Kim, B. Y. K., Lam, A. C. C., Phan, R. T., 1972. Druckverlust in kugelschttungen. Brennstoff-Warme-Kraft 24, 233-236.

Gupte, A., 1970. Experimentelle untersuchung der einflsse von porositt und korngrenverteilung im widerstandsgesetz der porenstrmung. Ph.D. thesis, Universitat Karlshruhe.

Leva, M., Grummer, M., 1947. Pressure drop through packed tubes, part iii, prediction of voids in packed tubes. Chem.Eng.Prog. 43, $713-718$.

Leva, M., Weintraub, M., Grummer, M., Storch, H. H., 1951. Fluid flow through packed and fluidized systems. Tech. Rep. 504, US Bureau of Mines. 
Mantle, M. D., Sederman, A. J., Gladden, L. F., 2001. Single and two-phase flow in fixed bed reactors: MRI flow visualisation and lattice-boltmann simulations. Chemical Engineering Science 56, 523 - 529.

Nakajo, S., Shigematsu, T., 2011. Experimental verification of turbulence modeling for the flow through a porous media by using ptv. In: Proceedings of 7th International Symposium on Turbulence and Shear Flow Phenomena (TSFP-7), Ottawa, Canada, July $28-31$.

Ookawara, S., Kuroki, M., Street, S., Ogawa, K., 2007. High-fidelity DEMCFD modeling of packed bed reactors for process intensification. In: Proceedings of European Congress of Chemical Engineering (ECCE-6), Copenhagen, 16-20th September.

Perry, R. H., Green, D. W., 1997. Perry's Chemical Engineers Handbook, 7th Edition. McGraw-Hill; New York.

Reichelt, W., 1972. Zur berechnung des druckverlustes einphasig durchstromter kugel- und zylinderschuttengen. Chemie-Ingenieur-Technik 44, 1068 - 1071.

Robbins, D. J., El-Bachir, M. S., Gladden, L. F., Cant, R. S., von Harbou, E., 2012. CFD modeling of single-phase flow in a packed bed with MRI validation. AIChE.J. 58 (12), $3904-3915$.

Sahimi, M., 1994. Applications of Percolation Theory. Taylor \& Francis, London.

Sullivan, S. P., Sana, F. M., Johns, M. L., Gladden, L. F., 2005. Simulation of packed bed reactors using lattice boltmann methods. Chemical Engineering Science 60, $3405-3418$.

Tóbis, J., 2000. Influence of bed geometry on its frictional resistance under turbulent flow conditions. Chem.Eng.Sci. 55, 5359 - 5366.

Tobis, J., 2002. Modeling of the pressure drop in the packing of complex geometry. Industrial and Engineering Chemistry Research 41, 2552 - 2559.

Tsotas, E., 2002. Fluid flow in packed beds. Chem.Eng.Sci. 20, $237-246$.

Yevseyev, A. R., Nakoryakov, V. E., Romanov, N. N., 1991. Experimental investigation of a turbulent filtrational flow. Int.J.Multiphase Flow 17 (1), $103-118$. 